\title{
Determination of diffractive parton densities at the LHeC and the FCC-eh
}

\author{
Anna M. Staśto* \\ Department of Physics, The Pennsylvania State University, University Park, PA 16802, U.S.A. \\ E-mail: ams52@psu.edu

\section{Nestor Armesto} \\ Instituto Galego de Física de Altas Enerxías IGFAE, Universidade de Santiago de Compostela, \\ 15782 Santiago de Compostela, Galicia-Spain \\ E-mail: nestor.armesto@usc.es
}

\section{Paul R. Newman}

School of Physics and Astronomy, University of Birmingham, UK

E-mail: Paul. Newman@cern.ch

\section{Wojciech Słomiński}

Institute of Physics, Jagiellonian University, Krakow, Poland

E-mail: wojtek.slominskieuj.edu.pl

The Large Hadron-electron Collider ( $\mathrm{LHeC}$ ) is a proposed upgrade of the LHC at CERN. An ERL will provide electrons to collide with the HL-LHC, HE-LHC and the FCC-hh proton and ion beams to achieve per nucleon centre-of-mass energies 1.3-3.5 (0.8-2.2) TeV and luminosities $10^{3} 4(33) \mathrm{cm}^{-2} \mathrm{~s}^{-1}$. These three configurations will enlarge the kinematic plane by more than one order of magnitude towards smaller $x$ and larger $Q^{2}$ than HERA, which translates into a range of available momentum fraction of the diffractive exchange with respect to the hadron down to $10^{-5}$ for a wide range of the momentum fraction of the parton with respect to the diffractive exchange. In this presentation we show the large possibilities that they offer for the measurement of the diffractive structure functions and subsequent determination of diffractive parton densities DPDFs in proton. For $e A$, we find that an extraction of the currently unmeasured nuclear DPDFs is possible with similar accuracy to that in $e p$.

XXVII International Workshop on Deep-Inelastic Scattering and Related Subjects - DIS2019 8-12 April, 2019

Torino, Italy

\footnotetext{
* Speaker.
} 


\section{Diffraction in Deep Inelastic Scattering}

Diffractive events in hadronic collisions are characterized by rapidity gaps - absence of any activity in parts of the detector. In deep inelastic lepton-hadron scattering, in a diffractive process, the hadron stays intact after collision or dissociates into a state with the hadron quantum numbers. At the HERA electron-proton collider about $10 \%$ events were diffractive.

The precise measurement of diffraction in DIS is of great importance for our understanding of the dynamics of strong interaction. Diffraction can provide information about confinement, since the interaction is mediated by the composite object which carries quantum numbers of the vacuum and the proton remains intact. It is also sensitive to the dynamics at low values of Bjorken $x$, and potentially to higher twists. It also can provide important information about the nuclear shadowing due to the Gribov relation between scattering on nuclei and diffraction in electron-proton scattering. It can also be used to check the range of validity of the factorization theorems in QCD.

Future electron-proton machines, like the Large Hadron electron Collider [1] and the Future Circular Collider [2] in electron-hadron mode will allow for the study of Deep Inelastic Scattering in a previously unexplored new kinematic regime. These machines have potential to reach very low values of Bjorken $x$ and will also allow to explore a very large range of scales, up to very high values of $Q^{2}$.

It is desirable to explore the capabilities of the new machines as far as the diffractive DIS processes are concerned. In this presentation we shall demonstrate the potential of the $\mathrm{LHeC}$ and FCC-eh as far as the inclusive diffraction is concerned. We determine the accessible kinematic range for diffractive processes of both machines. We also demonstrate the potential of both machines to constrain the diffractive parton distribution functions. Finally, a simulation of the diffractive pseudodata for $e A$ collisions for different scenarios of nuclear shadowing is performed. The details of this analysis can be found in [3].

\section{Diffractive kinematics and pseudodata for $\mathrm{LHeC}$ and FCC-eh machines}

The diffractive deep inelastic event depicted in Fig.1 (left plot) is characterized by the standard DIS variables: $Q^{2}$, the (minus) virtuality of the photon, $x$, the Bjorken variable, and $W^{2}$, the centre-of-mass photon-proton energy squared. In addition, there are variables characteristic to the diffractive DIS event: $t$ is the squared four-momentum transfer at the proton vertex, $\xi$ (alternatively denoted $x_{\mathbb{P}}$ ) can be interpreted as the momentum fraction of the 'diffractive exchange' with respect to the hadron, and $\beta$ (alternatively denoted $z$ ) is the momentum fraction of the parton with respect to the diffractive exchange. The two momentum fractions combine to give Bjorken- $x, x=\beta \xi$.

In the right plot in Figure 1 the accessible kinematic range in $\left(x, Q^{2}\right)$ is shown for three machines: HERA, LHeC and FCC-eh. The HERA data shown in this plot are from ZEUS-LRG [4], H1-LRG [5], HERA-FLPS [6]. For the LHeC design the range in $x$ is increased by a factor $\sim 20$ over HERA and the maximum available $Q^{2}$ by a factor $\sim 100$. The FCC-eh machine would further increase this range with respect to the LHeC by roughly one order of magnitude in both $x$ and $Q^{2}$. The experimental acceptance for the scattered electron is assumed to be $1^{\circ}$, which is essential to obtain good sensitivity to the low $x$ and low $Q^{2}$ region. 

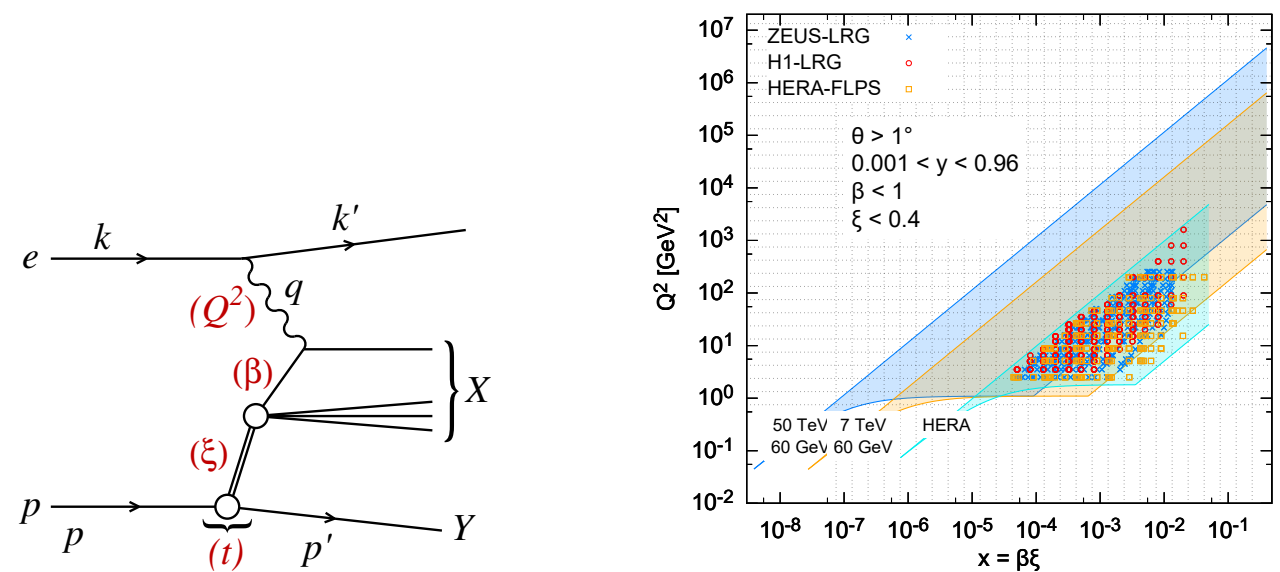

Figure 1: Left: A diagram of a diffractive neutral current event in DIS together with the corresponding variables, in the one-photon exchange approximation. The large rapidity gap is between the system $X$ and the scattered proton $Y$ (or its low mass excitation). Right: Kinematic phase space for inclusive diffraction in $\left(x, Q^{2}\right)$ for the LHeC (orange region) and the FCC-eh (blue region), compared with the HERA data (ZEUSLRG [4], H1-LRG [5] and HERA-FLPS [6]). The acceptance limit for the electron in the detector design has been assumed to be $1^{\circ}$.

Diffractive cross sections in the neutral current case, and integrated over the momentum transfer $t$, can be presented in the form of the reduced cross sections

$$
\frac{d^{3} \sigma^{\mathrm{D}}}{d \xi d \beta d Q^{2}}=\frac{2 \pi \alpha_{\mathrm{em}}^{2}}{\beta Q^{4}} Y_{+} \sigma_{\mathrm{r}}^{\mathrm{D}(3)}
$$

where $Y_{+}=1+(1-y)^{2}$.

The standard description of diffractive structure functions in perturbative QCD relies on collinear factorization [7]. Similarly to the inclusive case, the diffractive cross section can be factorized into the perturbatively calculable cross sections and the diffractive parton distribution functions DPDFs. The partonic cross sections are the same as in inclusive DIS. The DPDFs represent the probability distributions for partons in the proton under the constraint that the proton is scattered into the system with a specified 4-momentum. They obey the standard DGLAP evolution equations. The factorization theorem is valid for sufficiently high values of $Q^{2}$.

Fits to the diffractive structure functions were performed by both the H1 and ZEUS collaborations. In this analysis we use fit ZEUS-SJ [8]. Both types of fits parametrize the diffractive PDFs in a two component model, which is a sum of two exchange contributions, $\mathbb{P}$ and $\mathbb{R}$ :

$$
f_{i}^{\mathrm{D}(4)}\left(z, \xi, Q^{2}, t\right)=f_{\mathbb{P}}^{p}(\xi, t) f_{i}^{\mathbb{P}}\left(z, Q^{2}\right)+f_{\mathbb{R}}^{p}(\xi, t) f_{i}^{\mathbb{R}}\left(z, Q^{2}\right) .
$$

For both of these terms proton vertex factorization is assumed, meaning that the diffractive exchange can be interpreted as colourless objects called a 'Pomeron' or a 'Reggeon' with their corresponding parton distributions $f_{i}^{\mathbb{P}, \mathbb{R}}\left(\beta, Q^{2}\right)$. The flux factors $f_{\mathbb{P}, \mathbb{R}}^{p}(\xi, t)$ represent the probability that a Pomeron/Reggeon with given values $\xi$ couples to the proton. They are parametrized using a form motivated by Regge theory:

$$
f_{\mathbb{P}, \mathbb{R}}^{p}(\xi, t)=A_{\mathbb{P}, \mathbb{R}} \frac{e^{B_{\mathbb{P}, \mathbb{R}} t}}{\xi^{2 \alpha_{\mathbb{P}, \mathbb{R}}(t)-1}},
$$


with a linear trajectory $\alpha_{\mathbb{P}, \mathbb{R}}(t)=\alpha_{\mathbb{P}, \mathbb{R}}(0)+\alpha_{\mathbb{P}, \mathbb{R}}^{\prime} t$. Usually in the analysis one uses diffractive PDFs which are integrated over $t$

$$
f_{i}^{\mathrm{D}(3)}\left(z, \xi, Q^{2}\right)=\phi_{\mathbb{P}}^{p}(\xi) f_{i}^{\mathbb{P}}\left(z, Q^{2}\right)+\phi_{\mathbb{R}}^{p}(\xi) f_{i}^{\mathbb{R}}\left(z, Q^{2}\right) .
$$

where $\phi_{\mathbb{P}, \mathbb{R}}^{p}(\xi)=\int d t f_{\mathbb{P}, \mathbb{R}}^{p}(\xi, t)$.

The diffractive parton distributions of the Pomeron at the initial scale $\mu_{0}^{2}=1.8 \mathrm{GeV}^{2}$ are parametrized as

$$
z f_{i}^{\mathbb{P}}\left(z, \mu_{0}^{2}\right)=A_{i} z^{B_{i}}(1-z)^{C_{i}}
$$

where $i$ is a gluon or a light quark. In the diffractive parametrizations all the light quarks (antiquarks) are assumed to be equal. For the treatment of heavy flavours, a variable flavour number scheme (VFNS) is adopted and the parton distributions for the Reggeon component are taken from a parametrization which was obtained from fits to the pion structure functions.

Pseudodata for both $\mathrm{LHeC}$ and FCC-eh kinematic ranges were generated using the extrapolation of the fit ZEUS-SJ to HERA data, which provides the central values. The errors were simulated by performing a random Gaussian smearing with standard deviation corresponding to the relative error. An uncorrelated 5\% systematic error was assumed. The statistical error was computed assuming a very modest integrated luminosity of $2 \mathrm{fb}^{-1}$.

In Figure 2 we show a subset of the simulated data for the diffractive reduced cross section $\xi \sigma_{\mathrm{r}}$ as a function of $\beta$ in selected bins of $\xi$ and $Q^{2}$ for the LHeC (left plot) and FCC-eh (right plot) cases, respectively. For most data points the errors are very small, and are dominated by the systematics.
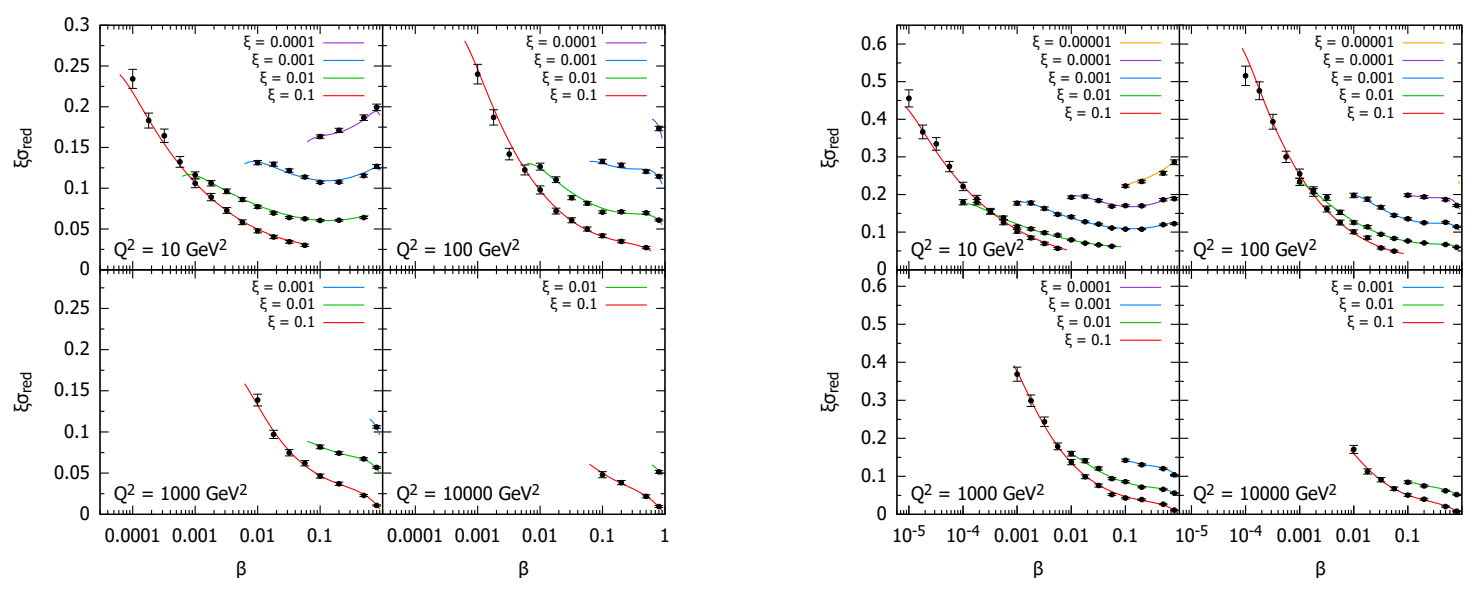

Figure 2: Selected subset of the simulated data for the diffractive reduced cross section as a function of $\beta$ in bins of $\xi$ and $Q^{2}$ for $e p$ collisions. Left: LHeC; right: FCC-eh.

Next, a study of the accuracy of extraction of the DPDFs was performed. In order to evaluate the precision with which the DPDFs can be determined, several pseudodata sets, corresponding to independent random error samples, were generated. Each pseudodata set was then fitted to the reduced cross-sections in the DPDF model corresponding to the same parametrization given by the ZEUS-SJ fit.

The minimal value of $Q^{2}$ for the data considered in the fits was set to $Q_{\min }^{2}=5 \mathrm{GeV}^{2}$ in order to avoid the sensitivity to the potential higher twists which may start to appear in this region. We 
also performed the study with lower values of $Q_{\min }^{2}$ and found reduction in the uncertainties of the DPDFs. This is connected with the fact that in the latter case more data points are included in the region which is closer to the initial scale of the evolution and, hence, they provide more constraints on the shape of the parametrization. It also indicates that both machines will have excellent potential to constrain the parametrization in the low $Q^{2}$ region and hence to unravel dynamics which is beyond the leading twist-2 DGLAP evolution.

The maximum value of $\xi$ was set by default to $\xi_{\max }=0.1$, above which the cross-section starts to be dominated by the Reggeon exchange. The region above the top threshold was not considered in the fits, and we found that the top contribution has a negligible impact for the $\mathrm{LHeC}$ but some impact for the FCC-eh. In Figures 3 the diffractive gluon distributions are shown for the LHeC, as a function of $z$ for fixed scales $\mu^{2}=6,20,60,200 \mathrm{GeV}^{2}$. The extrapolated ZEUS-SJ DPDFs are shown with error bands marked by the hatched area. On the other hand the bands labelled $A, B, C$ denote fits to three statistically independent pseudodata replicas, obtained from the same central values and statistic and systematic uncertainties. We see that the DPDFs determination accuracy improves with respect to HERA by a factor of 5-7 for the LHeC. A similar analysis for the FCC-eh shows an improvement of factor 10-15 over that at the HERA collider. We note that these uncertainties contain only those stemming from the experimental ones, but neither those of a theoretical origin (values of quark masses or of the strong coupling constant) nor those coming from the functional form of the initial distributions, Eq. (2.5).
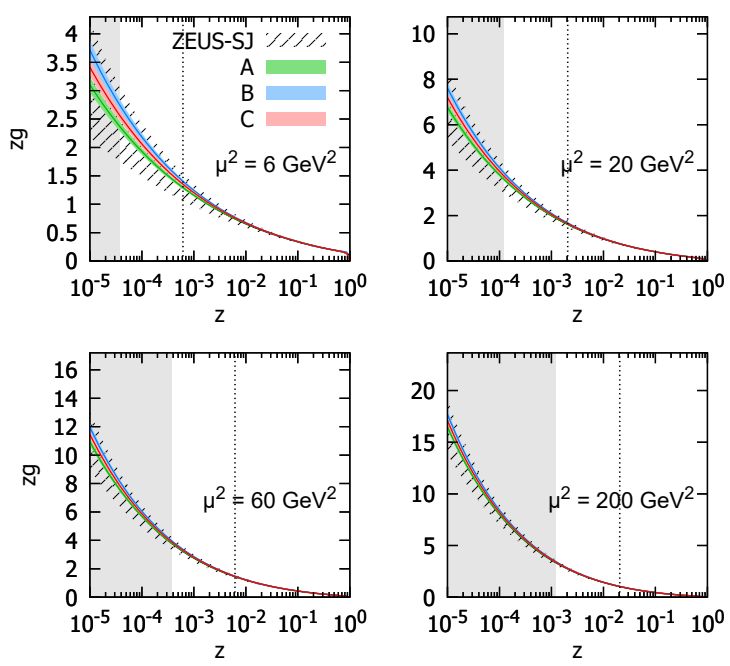

Figure 3: Diffractive PDFs for gluon in the LHeC kinematics as a function of momentum fraction $z$ for fixed values of scale $\mu^{2}$. Results of fits to three (A,B,C) pseudodata replicas are shown together with the experimental error bands. For comparison, the extrapolated ZEUS-SJ fit is also shown (black) with error bands marked with the hatched pattern. The vertical dotted lines indicate the HERA kinematic limit.

Electron-nucleus $(e \mathrm{~A})$ collisions are also possible at the LHeC and the FCC-eh with large integrated luminosities. In Fig. 4 pseudodata for the reduced cross section are shown for both machines. They were obtained using the same framework as in the proton case, and the model of [9] for the nuclear modification factor. 

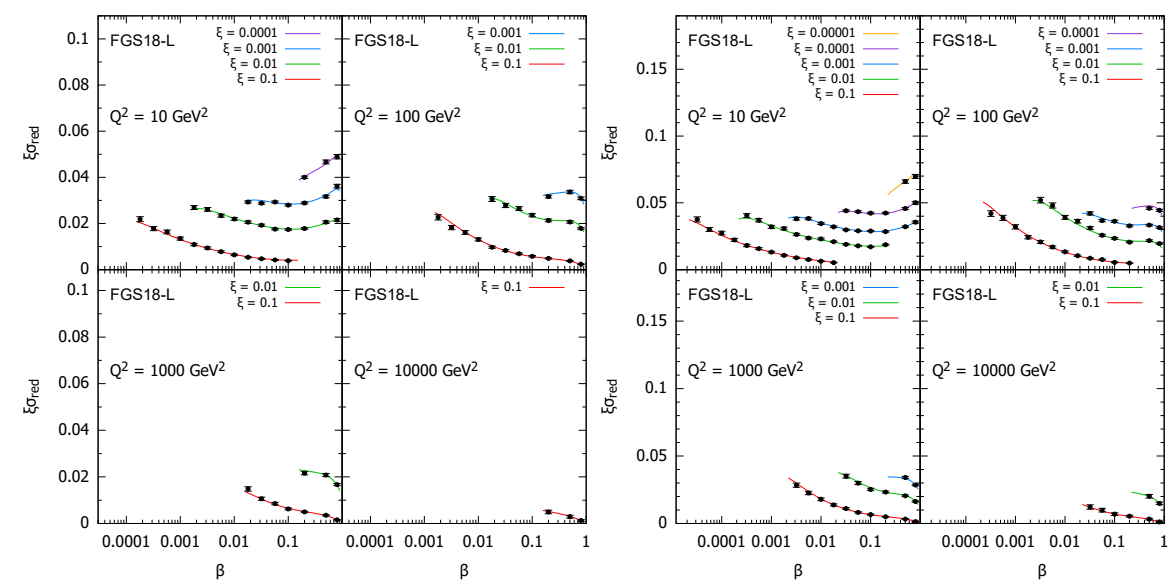

Figure 4: Simulated data for the diffractive reduced cross section as a function of $\beta$ in bins of $\xi$ and $Q^{2}$ for $e^{208} \mathrm{~Pb}$ collisions at the $\mathrm{LHeC}$ and at the $\mathrm{FCC}$-eh, in the models in [9].

Summarizing, both LHeC and FCC-eh machines offer an unprecedented potential for exploring the diffractive processes with excellent precision and in a completely new energy regime.

\section{Acknowledgements}

NA was supported by Ministerio de Ciencia e Innovación of Spain under projects FPA201458293-C2-1-P, FPA2017-83814-P and Unidad de Excelencia María de Maetzu under project MDM2016-0692, by Xunta de Galicia (Consellería de Educación) within the Strategic Unit AGRUP2015/11, and by FEDER. This work has been performed in the framework of COST Action CA15213 'Theory of hot matter and relativistic heavy-ion collisions' (THOR). WS was supported by the National Science centre, Poland, Grant No. 2014/13/B/ST2/02486. AMS was supported by the Department of Energy Grant No. DE-SC-0002145, as well as the National Science centre, Poland, Grant No. 2015/17/B/ST2/01838.

\section{References}

[1] J. L. Abelleira Fernandez et al. [LHeC Study Group], J. Phys. G 39, 075001 (2012).

[2] A. Abada et al. [FCC Collaboration], CERN-ACC-2018-0056.

[3] N. Armesto, P. R. Newman, W. Slominski and A. M. Stasto, arXiv:1901.09076 [hep-ph].

[4] S. Chekanov et al. [ZEUS Collaboration], Nucl. Phys. B 816, 1 (2009).

[5] F. D. Aaron et al. [H1 Collaboration], Eur. Phys. J. C 72, 2074 (2012).

[6] F. D. Aaron et al. [H1 and ZEUS Collaborations], Eur. Phys. J. C 72, 2175 (2012).

[7] J. C. Collins, Phys. Rev. D 57, 3051 (1998) Erratum: [Phys. Rev. D 61, 019902 (2000)].

[8] S. Chekanov et al. [ZEUS Collaboration], Nucl. Phys. B 831, 1 (2010).

[9] L. Frankfurt, V. Guzey and M. Strikman, Phys. Rept. 512, 255 (2012). 